\title{
PERCEPÇÕES DE URGÊNCIA PARA USUÁRIOS E MOTIVOS DE UTILIZAÇÃO DO SERVIÇO DE ATENDIMENTO PRÉ-HOSPITALAR MÓVEL
}

\author{
Kelly Piacheski de ABREU ${ }^{a}$, Alisia Helena Weis PELEGRINI ${ }^{\text {b }}$ \\ Giselda Quintana MARQUES ${ }^{c}$, Maria Alice Dias da Silva LIMA ${ }^{d}$
}

\section{RESUMO}

Os Serviços de Atendimento Móvel de Urgência atendem situações urgentes de diversas naturezas, e a procura ocorre conforme percepção do usuário sobre o que é urgente. O objetivo foi analisar percepções de urgência dos usuários que demandam atendimento no Serviço de Atendimento Móvel de Urgência de Porto Alegre e identificar motivos dessas solicitações. Estudo exploratório-descritivo, com abordagem qualitativa, com coleta de dados por meio de entrevistas semiestruturadas realizadas por telefone, no período de fevereiro a abril de 2009. Foi utilizada análise temática para tratamento das informações. Os usuários consideram como urgência condições que oferecem risco à vida, que necessitam de atendimento rápido, situações visíveis como sangramento, doenças crônicas e dificuldades de transporte e locomoção. Os usuários sentem-se motivados a acionar o serviço pela rapidez, pela gratuidade e para obter transporte. As percepções dos usuários sobre urgência são condizentes com a perspectiva biomédica dos profissionais de saúde e próprias do contexto social.

Descritores: Serviços médicos de emergência. Socorro de urgência. Necessidades e demandas de serviços de saúde. Assistência pré-hospitalar.

\section{RESUMEN}

Los Servicios de Atención Móvil de Urgencias atienden situaciones urgentes de diversa naturaleza y la solicitud de tal servicio corresponde con la percepción del usuario sobre lo que es urgente. El objetivo fue analizar las percepciones de urgencia de usuarios que solicitan atención en un Servicio de Atención Móvil de Urgencias de Porto Alegre e identificar los motivos referidos. Estudio exploratorio-descriptivo con enfoque cualitativo. Los datos fueron recolectados mediante entrevistas semiestructuradas realizadas telefónicamente, durante el período comprendido entre febrero y abril de 2009. Se utilizó Análisis Temático para tratamiento de la información. Los usuarios consideran urgentes condiciones que ofrecen riesgo para la vida, que necesitan de pronta atención, situaciones visibles como sangrado, enfermedades crónicas y dificultades de transporte y locomoción. Los usuarios prefieren el servicio por la rapidez, gratuidad y para obtener transporte. Las percepciones de usuarios sobre urgencias son diversas, algunas veces concordantes con la perspectiva biomédica del profesional de salud; otras, propias del contexto social.

Descriptores: Servicios médicos de urgencia. Socorro de urgencia. Necesidades y demandas de servicios de salud. Atención prehospitalaria.

Título: Percepciones de urgencia para usuarios y motivos de utilización del servicio de atención prehospitalaria móvil.

\section{ABSTRACT}

The Mobile Emergency Care Services handle urgent situations of various types, and demand for this service occurs according to the perception of the user regarding what is urgent. The aim of the study was to analyze the perceptions of urgency by users who demand care from a Mobile Emergency Care Service in Porto Alegre and to identify the reasons for these requests. In this exploratory-descriptive study, with qualitative approach, data were collected through semi-structured interviews conducted by telephone, during the period from February to April 2009. Thematic analysis was used to analyze the information. Users consider urgent life-threatening conditions, which require a quick response, and visible situations, such as bleeding, chronic diseases, and difficulties in transportation. Users themselves feel motivated to call the service due to it being rapid and free, and for transportation. The perceptions of users regarding urgency were diverse, sometimes consistent with the biomedical perspective of health providers and sometimes with their own social context.

Descriptors: Emergency medical services. Emergency relief. Health services needs and demands. Pre-hospital Care Title: Users' perceptions of urgency and reasons for using the mobile pre-hospital care service.

a Enfermeira, Mestranda pelo Programa de Pós-Graduação em Enfermagem da Universidade Federal do Rio Grande do Sul (PPGENF / UFRGS), Porto Alegre, Rio Grande do Sul, Brasil.

b Enfermeira, Doutoranda pelo PPGENF / UFRGS, Bolsista do Programa de Apoio a Planos de Reestruturação e Expansão das Universidades Federais (REUNI), Porto Alegre, Rio Grande do Sul, Brasil.

c Doutora em Enfermagem pelo PPGENF / UFRGS, Enfermeira da Secretaria Municipal de Saúde, Porto Alegre, Rio Grande do Sul, Brasil.

d Enfermeira, Doutora em Enfermagem, Professora do PPGENF / UFRGS, Pesquisadora do Conselho Nacional de Desenvolvimento Científico e Tecnológico (CNPq), Porto Alegre, Rio Grande do Sul, Brasil. 


\section{INTRODUÇÃO}

A percepção de sinais e sintomas como urgentes é um fator decisivo para a utilização dos serviços de saúde, pois é a partir dela que os usuários elaboram mecanismos e ações para recuperação da saúde ${ }^{(1)}$. A escolha do serviço a ser utilizado se dará conforme a percepção do usuário do que é simples ou grave, bem como pela possibilidade de acesso e capacidade resolutiva do serviço cogitado para atendimento ${ }^{(2)}$.

Os usuários buscam serviços que ofereçam vantagens que podem estar relacionadas aos horários de atendimento, à qualidade da oferta, assim como à distância percorrida, uso de meios de transporte e tempo decorrido até o serviço ${ }^{(3)}$. Nas situações consideradas urgentes o fator tempo é determinante da tomada de decisão de qual serviço deva ser usado, modulando o tipo de demanda e o serviço a ser consumido ${ }^{(1)}$.

Neste contexto, os serviços pré-hospitalares móveis de urgência se apresentam à população como possibilidade de acesso rápido e eficaz, visto que possibilitam cuidados na cena, interface com diferentes serviços de saúde, bem como podem regular o acesso do usuário ao sistema de saúde ${ }^{(4)}$.

Os chamados feitos ao Serviço de Atendimento Móvel de Urgência (SAMU) são acolhidos e avaliados pela Central de Regulação Médica. Telefonistas auxiliares de regulação médica atendem a chamada, coletam informações de identificação e transferem a ligação para os médicos reguladores, que presumem a gravidade da situação e definem o tipo de atenção que será oferecido ${ }^{(4)}$. Quando os médicos reguladores avaliam que a demanda não condiz com as possibilidades de atendimento pelo SAMU, definem o chamado como não pertinente. Nesses casos, prestam informações ao telefone, orientam cuidados ou indicam serviços de saúde.

Dentre as solicitações recebidas pela Central de Regulação em 2008, 33\% foram classificadas como situações não pertinentes à finalidade do SAMU e $17,3 \%$ não possuíam dados suficientes para tomada de decisão do médico regulador ${ }^{(5)}$. As demandas não pertinentes dizem respeito à percepção do solicitante de um quadro de saúde identificado como urgente, mas que não se enquadra nos critérios de gravidade e prioridade estabelecidos pela Central de Regulação Médica. Em algumas situações, a procura é considerada inadequada pelos profissionais da saúde, o que tem gerado conflito de interesses ${ }^{(5-6)}$.
Dessa forma, questiona-se: O que é considerado como situação urgente para os usuários que demandam atendimento do SAMU? Quais são os motivos para utilização desse serviço? A percepção que os usuários têm sobre as situações urgentes pode auxiliar os profissionais da saúde no reconhecimento de demandas e no planejamento de ações que beneficiem usuários por meio da interface que o SAMU estabelece com os serviços de saúde.

A compreensão das percepções dos usuários sobre as situações de urgência produz conhecimento que pode ser utilizado na formulação de novas hipóteses sobre os motivos que influenciam a busca pelos serviços pré-hospitalares móveis. Entender essa utilização pode servir de ferramenta na organização dos serviços que atendem agravos urgentes e contribuir para ampliar o acesso aos acometidos por agravos de menor gravidade. Permite, também, à enfermagem, compreender as demandas dos usuários e atuar sobre elas, por meio de orientações e cuidados qualificados.

Neste artigo, que se originou de monografia de conclusão de curso de graduação em enfermagem $^{(6)}$, tem-se por objetivo analisar a percepção de urgência dos usuários que demandam atendimento ao SAMU de Porto Alegre e identificar os motivos dessas solicitações.

\section{METODOLOGIA}

Trata-se de pesquisa exploratório-descritiva com abordagem qualitativa. A coleta de dados foi realizada no SAMU de Porto Alegre, RS, Brasil. Realizou-se entrevista semi-estruturada por telefone para coleta dos $\operatorname{dados}^{(7)}$.

Para a seleção dos participantes foi usado um recorte de tempo de 24 horas na base de dados do SAMU. Realizou-se um sorteio dos participantes na listagem de demandas do dia, para seleção de trinta usuários, maiores de 18 anos, que solicitaram atendimento ao SAMU, sendo quinze participantes com chamados classificados como pertinentes ao serviço e quinze, não pertinentes. As ligações originadas dos telefones de via pública foram descartadas, uma vez que era difícil localizar o usuário por meio desse número telefônico.

Após o sorteio dos participantes, procedeu-se a impressão das fichas de regulação em que constavam dados de identificação e de atendimento dos usuários. O número de indivíduos entrevistados foi estabelecido levando em consideração o princípio de saturação de dados ${ }^{(8)}$. 
As entrevistas foram realizadas no período de 11 de fevereiro a 30 de abril de 2009. Estabeleceu-se contato por telefone com os usuários sorteados para a coleta de dados. Seguiu-se um roteiro com questões sobre o que consideram urgência e sobre os motivos pelos quais tinham acionado o SAMU. As entrevistas foram gravadas e transcritas na íntegra, sendo codificadas com letras e números: P1 a P30.

Para o tratamento dos dados coletados foi utilizada a técnica de análise de conteúdo, do tipo análise temática ${ }^{(10)}$.

O estudo está inserido no projeto intitulado "Epidemiologia, territorialização e organização do trabalho no atendimento pré-hospitalar móvel de Porto Alegre", aprovado pelo Comitê de Ética em Pesquisa da Secretaria Municipal de Saúde (Parecer $\mathrm{n}^{\circ}$ 117/2006). Seguindo as recomendações da Resolução 196/96 do Conselho Nacional de Saúde ${ }^{(9)}$, por se tratar de entrevista por telefone, o Termo de Consentimento Livre e Esclarecido foi substituído pelo consentimento tácito dos participantes, gravado no momento do contato telefônico.

\section{RESULTADOS}

Os resultados indicam diferentes percepções sobre as situações de urgência, que variam de acordo com os problemas de saúde do usuário. Assim, as situações de urgência podem estar relacionadas à necessidade de atendimento imediato.

Eu acho que a parte de urgência é quando o atendimento tem que ser de imediato. Tu solicitas a emergência e ela tem um tempo mínimo para fazer o atendimento necessário. (P O1)

Outros acreditam que urgência é tanto dificuldade de mobilização quando de transporte até um serviço de saúde:

Quando a pessoa está totalmente debilitada, que não pode caminhar ou se mover. ( $\mathrm{P} 07$ )

Citam, também, as situações ameaçadoras à vida, com sinais e sintomas sugestivos de gravidade.

Tipo um corte muito profundo, bater a cabeça muito forte, uma coisa que tu não podes esperar uma conduta em um posto de saúde ou que tu possas te locomover com onibus e que te leve a um pronto-socorro[...] ( $\mathrm{P}$ O2)
As percepções podem se relacionar a tudo que não está bom, nesse sentido, qualquer situação pode ser uma urgência que demanda atendimento.

\section{Qualquer caso, qualquer estado clínico, qualquer pessoa} que estiver se sentindo ruim. (P 15)

Alguns sinais e sintomas de aparecimento súbito são definidos como urgentes, indicando a necessidade de avaliação imediata por profissionais de saúde, visto que para o solicitante são sinais preocupantes.

Começou a sair sangue pelo nariz dele [...] Assim, para a idade dele eu acho que seria quebrar alguma coisa, fratura exposta, com sangue, eu acho que com sangue é mais urgente. (P 17)

As demandas ao serviço são formuladas a partir da percepção do solicitante. Por vezes, a concepção da urgência é distinta na visão de usuários e de profissionais de saúde. Para usuários está articulada ao contex to geral no qual o sintoma ou o problema de saúde se manifesta e para profissionais é definida com base na gravidade dos casos e no saber biomédico.

Olha, que nem o caso do meu sobrinho que estava tendo uma crise de asma ou alguma coisa assim. Era grave e o médico disse que não era grave, que não era urgência. (P 18)

Alterações visíveis no estado de saúde, como a presença de sangue e a perda da consciência, geram maior mobilização por parte da população. Embora muitas condições não se configurem como situações de urgência e não haja necessidade do envio de equipes de socorro, demonstram o interesse dos usuários na avaliação por profissionais de saúde.

\section{Eu procurei numa situação que me dissessem o que fazer, porque a parte de primeiros socorros a gente não tem muita base. (P O3)}

O solicitante pode não estar esclarecido sobre qual serviço de saúde deva utilizar, buscando atendimento no local que identifica como sendo o mais adequado.

A gente utiliza o SAMU como um meio de esclarecimento do que fazer [...] Ligar para pedir orientação para o médico, eu acho que isso é uma coisa boa, se as outras 
pessoas tivessem pelo menos essa coisa de [...] procurar a orientação antes de tomar uma atitude. (P 16)

As demandas estão vinculadas a critérios individuais ou específicos de grupos sociais que, por meio da percepção da urgência, definem o que será buscado para o atendimento do usuário.

Os solicitantes ajustam a oferta de serviços do SAMU ao seu contex to vital, familiar ou de trabalho, estruturando, a partir daí, suas demandas. Com base nessa afirmação são explicitados os motivos pelos quais os participantes procuram atendimento no SAMU.

A busca pelo serviço é motivada pela rapidez e presteza no atendimento oferecido, sendo capaz de transportar o usuário para um serviço que corresponda à necessidade de saúde da pessoa.

A gente estava apavorada, não sabia o que era e ele estava ruim, a gente achou que a SAMU era o mais rápido para chegar alipara atender ele e era muito tarde da madrugada. Ele começou a se sentir mal e eu achei que seria o mais rápido para vir buscar ele, atender, de repente, alguém ali para dizer se precisava levar para o hospital ou não. (P18)

Outro motivo de solicitação identificado foi pela necessidade de um meio de transporte para deslocamento até o serviço de saúde, visto que o solicitante não dispunha de recurso próprio para realizar a transferência.

Não tinha como locomover ele [...] o nosso maior problema [para uma Escola] é quando a gente não tem como levar para algum lugar rápido, então o SAMU. [...] E nós nos deparamos diariamente com casos que o pai e a mãe diz que não tem condições de levar o filho, não tem dinheiro para a passagem. (P 16)

Dificuldades financeiras também estão dentre os motivos da procura pelo SAMU, especialmente para os mais pobres, sendo que o custo do deslocamento é decisivo na procura pelo serviço.

A forma como está organizado o atendimento com um interlocutor permanente, a disponibilidade de equipes qualificadas para o atendimento de urgência, também motiva as demandas, o que dá ao serviço a credibilidade necessária para o atendimento propriamente dito ou para fornecer informações e orientações ao telefone.

Chamei pelo motivo do médico estar falando comigo ao mesmo tempo e estar me orientando para o que deveria fazer [...] É um médico, por mais que ele esteja do outro lado da linha, tem experiência nisso, se fosse alguma coisa mais grave eu acho que eles teriam vindo. Então aí ele me tranquilizou, dizendo o que eu devia fazer. (P17)

Nas instituições onde não há médico disponível para atendimento de situações agudas, como no caso de clínicas geriátricas, o SAMU tem servido de apoio aos profissionais.

Porque aqui na Clínica geriátrica a gente é orientado a chamar a SAMU quando os idosos estão com os sinais vitais alterados. (P8)

Aliada a todos os motivos citados, a facilidade em obter a ligação pelo número 192, dá às pessoas a possibilidade de se comunicar com a Central de Regulação, mesmo naquelas situações em que o solicitante desconhece a pessoa que demanda atendimento.

Eu achei que era da competência do SAMU [remover morador de rua]. E daí junto com mais outras pessoas nós ligamos ao SAMU. Era o único número de telefone que eu tenho cadastrado no meu celular. (P O4)

Situações em que pacientes apresentam desestabilização de quadros crônicos, como por exemplo, hipoglicemia, crise hipertensiva, distúrbios psiquiátricos, entre outros, também são geradoras de demandas.

Eu seguido uso a ambulância [...] para minha mãe que seguido dá hipoglicemia. Ela anda tomando os remédios errado. (P6).

A utilização dos serviços de urgência como principal fonte de cuidados pode ocasionar progressão de complicações, ocasionando prejuízo ao doente, uma vez que esses serviços não propiciam a continuidade da assistência. Muitos usuários que buscam atendimento no SAMU se beneficiariam dos serviços disponíveis na Atenção Primária à Saúde, pois poderiam usufruir de uma melhor abordagem do problema de saúde apresentado por meio da continuidade do tratamento, prevenindo a piora do caso.

\section{DISCUSSÃO}

As percepções dos usuários sobre urgência são diversas e encontram-se vinculadas aos problemas e às necessidades de saúde que compreendem desde 
o risco à vida até situações de menor complexidade e gravidade. Esses aspectos indicam que a percepção de urgência está alicerçada em situações, nem sempre condizentes às manifestações biológicas de maior gravidade, mas que estão interligadas a critérios e necessidades de quem solicita atendimento ${ }^{(11)}$.

O usuário recorre ao SAMU com a intenção de resolver seus problemas de saúde que julga urgente, e utiliza comportamentos e experiências individuais e coletivas para classificá-los como tal e a partir disso, solicitar atendimento. A percepção de sintomas como perda de saúde gera ansiedade, em especial quando o usuário desconhece o que possa estar ocorrendo, sendo assim, a interlocução com um profissional da saúde auxilia na tomada de decisão e lhe dá segurança ${ }^{(1)}$.

Já foi identificado que o contato do usuário com um profissional de saúde, na Central de Regulação do SAMU ou na cena, com uma equipe de socorro, possibilita uma interação que propicia o compartilhamento de responsabilidades frente ao desconhecimento do que pode e/ou deve ser feito nas situações percebidas como urgentes ${ }^{(4)}$. Para outro autor ${ }^{(12)}$, a busca de um serviço de ambulâncias deriva da percepção do usuário de estar doente, além do que é um meio seguro e confiável para receber atendimento, representando uma possibilidade de acesso facilitado aos serviços hospitalares.

Estudo realizado em oficinas de primeiros socorros identificou que usuários, apesar de desconhecerem aspectos inerentes à atuação em situações de urgência, conheciam a finalidade do SAMU, bem como os procedimentos necessários para solicitá-lo, reconhecendo-o como meio de transporte ao pronto atendimento das demandas. As autoras atribuíram esse fato à provável dificuldade no acesso aos demais serviços de saúde ${ }^{(13)}$.

A procura espontânea por atendimento a uma situação de baixa gravidade ou que não corresponda à finalidade dos serviços pré-hospitalares móveis de urgência é um fenômeno que não ocorre só no Brasil. O SAMU 15, de Paris, França, no período de 10 anos, atendeu a um número significativo de chamados que poderiam ser caracterizados como missões de bom samaritano. As reais urgências representaram em torno de $6 \%$ do total de demandas atendidas. Foi identificado que as pessoas que imigravam, as que estavam expostas às situações de violência, ao medo e à insegurança e as que não dispunham de um médico de família ou pessoa que pudesse dispensar um conselho, ensinamento ou cuidado, desenvolviam sintomas e sinais pouco claros do ponto de vista médico. Essas questões não resolvidas aumentavam o consumo de serviços de saúde, especialmente os da urgência ${ }^{(14)}$.

Na Suécia, a utilização do serviço pré-hospitalar móvel por pacientes com disfunção vital importante é de $10 \%$ a $20 \%$ dos atendimentos ${ }^{(15)}$. A maioria dos pacientes é transportada para um serviço de emergência por ambulância totalmente equipada, sem requerer, no entanto, cuidados pré-hospitalares, seja na cena ou no transporte. Em 55\% dos casos o usuário poderia chegar ao hospital no próprio carro ou de taxi. No entanto, para os pacientes, nenhum veículo é tão bem equipado ou seguro quanto uma ambulância ${ }^{(16)}$.

Isso significa que um percentual menor de pacientes que são atendidos e transportados pelas unidades pré-hospitalares móveis precisa de cuidados de maior complexidade tecnológica, mas que a necessidade de recorrer à utilização de ambulâncias pelo usuário deriva da sua percepção do problema de saúde, da segurança e confiabilidade no serviço, além da possibilidade de acesso facilitado aos serviços hospitalares e de pronto atendimento.

$\mathrm{O}$ acesso às portas da urgência é um fato já identificado no SAMU de Porto Alegre, visto que $72,3 \%$ dos usuários atendidos por situações clínicas foram transportados para um serviço de saúde, sendo $49,4 \%$ levados para hospitais e $20 \%$ para unidades de pronto atendimento. Dentre esses atendimentos, apenas 3,7\% dos usuários eram acometidos por urgências de gravidade severa ${ }^{(4)}$. Isso pode significar que o usuário, ao identificar no seu estado de saúde a necessidade de cuidados de um profissional da saúde, reconhece no SAMU uma possibilidade de atendimento e de ser transportado para um serviço de urgência.

Neste estudo é possível destacar a função assistencial e social do SAMU, visto que sua intervenção orienta e informa o usuário, antecipa cuidados pelas equipes de socorro e estabelece diagnóstico precocemente, tendo resolutividade na cena ou removendo o usuário para uma porta da urgência de forma segura e ágil, o que é reconhecido pelos usuários.

Historicamente, os serviços de ambulâncias têm sido vistos como responsáveis pelo transporte de pessoas doentes para os hospitais. A concepção de ambulâncias equipadas com recursos tecnológicos avançados e capazes de estabilizar o paciente, na cena, é recente ${ }^{(15)}$. Assim, a ambulância é vista pela população como um veículo que transporta pacientes de um lugar para outro de forma segura. 
$\mathrm{Na}$ memória de brasileiros que viveram nas décadas de 1950 e 1960, o SAMU relembra o Serviço de Assistência Médica Domiciliar de Urgência (SAMDU), que se dispunha a prestar assistência médica no domicílio do usuário ${ }^{(4)}$. Do ponto de vista histórico, guardando as devidas proporções, o SAMU pode ser considerado como um embrião do Atendimento Pré-Hospitalar (APH) móvel, no Brasil, que precisa se desenvolver. Na visão dos usuários, a finalidade desse serviço precisa ser ampliada e qualificada.

\section{CONCLUSÕES}

As percepções dos usuários sobre urgência são variadas e se encontram vinculadas a problemas e necessidades de saúde que imprimem risco à vida, que precisam de atendimento imediato. Entretanto, essas percepções nem sempre correspondem às alterações biológicas consideradas urgentes pelos critérios biomédicos.

O fato dos serviços de atendimento pré-hospitalar móvel oferecerem avaliação e cuidados na cena, aliados ao transporte do paciente aos serviços de urgência, faz com que a população os veja como uma modalidade de atendimento, capaz de responder às suas solicitações, independentemente da gravidade da situação. Dessa forma, os usuários consideram o SAMU um serviço de credibilidade e confiança.

A utilização dos serviços de urgência pelo usuário é uma questão que gera polêmica entre alguns pesquisadores e profissionais da saúde, principalmente quando se refere às situações por eles classificadas como não urgentes. Para os primeiros, esse uso inadequado faz com que os serviços de urgência realizem atividades que não demandariam a necessidade de atendimento pré-hospitalar móvel. Para os usuários dizem respeito à subjetividade e aos sentidos atribuídos para sinais e sintomas de algo que não vai bem, sendo influenciada pelos aspectos culturais e sociais de cada pessoa ou região, da necessidade de atenção imediata que depende, tanto do ponto de vista do doente, quanto das estruturas assistenciais e a finalidade de cada serviço.

Dentro das opções que se apresentam no sistema de saúde, a escolha do usuário pelos serviços de urgência está fundamentada na expectativa de uma atenção rápida, segura e de maior confiança. A busca pela atenção imediata traz consigo o modelo biomédico, construído ao longo do tempo como uma prática social que tem privilegiado ações curativas, individuais, hospitalocêntricas, com intervenções medicalizantes.

Há que se considerar, ainda, a crescente medicalização da vida, fazendo com que se consuma número excessivo de medicamentos e torne a população cada vez mais dependente dos profissionais e dos serviços de saúde. A mudança desse paradigma não ocorrerá unilateralmente e exige esforço concentrado de vários atores sociais.

Apesar das transformações no modelo de atenção à saúde, com investimentos em vários âmbitos, a esfera da atenção hospitalar ainda carrega a marca da resolutividade. É por esse atendimento de qualidade que a população procura quando necessita de atenção que considera urgente.

A interpretação da necessidade como sendo de atenção imediata é um determinante de uso de serviços de urgência documentado na literatura. Sendo assim, é difícil educar a população para que não procure os serviços de urgência se o caso não for grave, quando são poucos os recursos ofertados para resolução de situações agudas que se apresentam no cotidiano. Também não pode ser esquecido que é o usuário quem define o que usar e onde usar, com base nas suas experiências, conhecimento da oferta de serviços, condição vital e do contexto social em que vive.

A principal limitação deste estudo se relaciona ao fato de que o desenho metodológico utilizado não permite a realização de associação entre aspectos de utilização do SAMU pelos usuários classificados como pertinentes ou não pertinentes na Regulação Médica com os motivos para acionar o serviço e a percepção de urgência dos grupos. Pesquisas sobre essa temática são necessárias, visto a escassez de estudos realizados sob a perspectiva dos usuários nos serviços de atendimento pré-hospitalar móveis. Sugere-se a realização de estudos de associação entre utilização do atendimento pré-hospitalar móvel por usuários não pertinentes e a utilização dos demais serviços de saúde disponíveis no sistema de saúde.

\section{REFERÊNCIAS}

1 Pasarín MI, Sanmamed MJF, Calafell C, Rodríguez D, Campasol S, Torné E, et al. Razones para acudir a los servicios de urgencias hospitalarios. La población opina. Gac Sanit. 2006;20(2):91-100.

2 Marques GQ, Lima MADS. Demandas de usuários a um serviço de pronto atendimento e seu acolhi- 
mento ao sistema de saúde. Rev Latino-Am Enferm. 2007;15(1):13-9.

3 Dall'Agnol CM, Lima MADS, Ramos DD. Fatores que interferem no acesso de usuários a um ambulatório básico de saúde. Rev Eletr Enf. 2009;1 1(3):674-80.

4 Marques GQ. Acesso e utilização do serviço de atendimento móvel de urgência de Porto Alegre pelos usuários com demandas clínicas [tese]. Porto Alegre: Escola de Enfermagem. Universidade Federal do Rio Grande do Sul; 2010.

5 Ciconet RM. Equipes de suporte básico do SAMU-192 de Porto Alegre: atuação e articulação das ações com a central de regulação e as portas de entrada da urgência. [dissertação]. Porto Alegre: Escola de Enfermagem, Universidade Federal do Rio Grande do Sul; 2009.

6 Abreu KP. Situações de urgência: visão dos usuários do Serviço de Atendimento Móvel de Urgência de Porto Alegre [trabalho de conclusão de curso]. Porto Alegre: Universidade Federal do Rio Grande do Sul. Escola de Enfermagem; 2009.

7 Musselwhite K, Cuff L, MacGregor L, King KM. The telephone interview is an effective method of date collection in clinical nursing research. Int J Nurs Stud. 2007;44(6):1064-70.

8 Fontanella BJB, Rica J, Turato ER. Amostragem por saturação em pesquisas qualitativas em saúde: contribuições teóricas. Cad Saúde Pública. 2008;24(1):17-27.
9 Ministério da Saúde (BR), Conselho Nacional de Saúde, Comitê Nacional de Ética em Pesquisa em Seres Humanos. Resolução 196, de 10 de outubro de 1996: diretrizes e normas regulamentadoras de pesquisa envolvendo seres humanos. Brasília (DF); 1997.

10 Bardin L. Análise de conteúdo. Lisboa: Edições 70; 2004 .

11 Giglio-Jacquemot A. Urgências e emergências em saúde: perspectivas de profissionais e usuários. Rio de Janeiro: Fiocruz; 2005.

12 Ahl C, Nyström M, Jansson L. Making up one's mind: - patients' experiences of calling an ambulance. Acc Emerg Nurs. 2006;14:11-19.

13 Veronese AM, Oliveira DLLC, Rosa IM, Nast K. Oficina de primeiros socorros: relato de experiências. Rev Gaúcha Enferm. 2010;31(1):179-82.

14 Barrier G. Les appels d'urgence au SAMU. C.R. Acad. Sci. Paris. 2001;324(7):663-666.

15 Suserud B-O. A new profession in the pre-hospital care field - the ambulance nurse. Nurs in Crit Care. 2005;10(6):269-71.

16 Hjalte L, Suserud B-O, Herlitz J, Karlberg I. Why are people without medical needs transported by ambulance? A study of conditions for pre-hospital care. Eur J Emerg Med. 2007;14(3):15-6.

\author{
Endereço do autor / Dirección del autor / \\ Author's address: \\ Kelly Piacheski de Abreu \\ Rua Gen. Couto de Magalhães, 1912, ap. 302, São João \\ 90540-130, Porto Alegre, RS \\ E-mail: kelly.piacheski@gmail.com
}

